\title{
Rapid and Durable Response With Nab-Sirolimus After Everolimus Failure in a Patient With Perivascular Epithelioid Cell Tumors (PEComas) of the Uterus
}

\author{
Pallavi Kopparthy ${ }^{1}$, Martina Murphy ${ }^{2}$ \\ 1. Medicine, University of Florida, Gainesville, USA 2. Hematology and Oncology, University of Florida Health, \\ Gainesville, USA
}

Corresponding author: Pallavi Kopparthy, kopparthy.pallavi@gmail.com

\begin{abstract}
Perivascular epithelioid cell tumors (PEComas) are rare mesenchymal tumors with a natural history ranging from indolent benign lesions to ones with an aggressive clinical course including distant metastases. Recent reports have suggested that mTOR inhibitor sirolimus and related drugs show some benefit in non-tuberous sclerosis complex PEComas. However, therapeutic options for patients who progress on sirolimus are very limited. We describe a patient with metastatic uterine PEComa, who progressed on mTOR inhibitor everolimus but had a rapid and durable response to nab-sirolimus.
\end{abstract}

Categories: Oncology

Keywords: gynecological cancer, pharmacology and therapeutics, pecoma

\section{Introduction}

Perivascular epithelioid cell tumors (PEComas) are rare mesenchymal tumors that show immunophenotypic features of smooth muscle and melanocytic differentiation. The term PEComa was first used in 1996 to designate neoplasms containing epithelioid cells with a close association to vessel walls and recently recognized as a distinct entity by the World Health Organization [1]. They include tumors like renal angiomyolipoma, pulmonary lymphangioleiomyomatosis (LAM), which are relatively benign lesions and seen in patients with tuberous sclerosis complex (TSC). However, the spectrum also includes rarer tumors of variable malignant potential involving lung, gynecologic and gastrointestinal systems; the latter not common in TSC [2].

Review began 04/13/2021 Review ended 05/10/2021 Published 05/11/2021

(c) Copyright 2021

Kopparthy et al. This is an open access article distributed under the terms of the Creative Commons Attribution License CC-BY 4.0., which permits unrestricted use, distribution, and reproduction in any medium, provided the original author and source are credited.
Evidence from a registration trial in advanced malignant PEComa (AMPECT) suggests that patients with AMPECT with no prior exposure to mTOR inhibitors, particularly those with TSC1 or TSC2 mutations, benefit from the use of an investigational mTOR inhibitor, nab-sirolimus (also known as albumin-bound sirolimus nanoparticles and ABI-009) [3]. An expanded access protocol (EAP) bridging between the closure of the AMPECT study and the marketing approval of the product gives patients with serious condition access to treatment with nab-sirolimus. AMPECT patients with and without prior exposure to mTOR inhibitors, other than nab-sirolimus were included. Here we report a case of metastatic uterine PEComa enrolled in the EAP, who progressed on the mTOR inhibitor everolimus yet had a rapid and durable response to nabsirolimus.

\section{Case Presentation}

\section{Diagnosis}

A 58-year-old post-menopausal female presented with abnormal uterine bleeding. Endometrial biopsy revealed a neoplastic process and further workup with a CT scan showed a $7 \mathrm{~cm}$ mass in the uterus. No other metastatic lesions were found. Following this, a laparoscopic hysterectomy with bilateral salpingooophorectomy was performed and pathology was consistent with malignant PEComa, which stained positive for smooth muscle actin, HMB-45, and Melan-A (59 mitoses per 10hpf). Foundation one genomic testing was positive for TSC1 with stable microsatellite status and low tumor mutation burden.

\section{Treatment history}

Chemotherapy

The primary tumor was locally advanced at diagnosis, adjuvant chemotherapy was not administered, and the patient was monitored with serial scans. CT scan at six months following surgery showed multiple bilateral pulmonary nodules, consistent with metastatic disease (Figure 1). 


\section{Cureus}

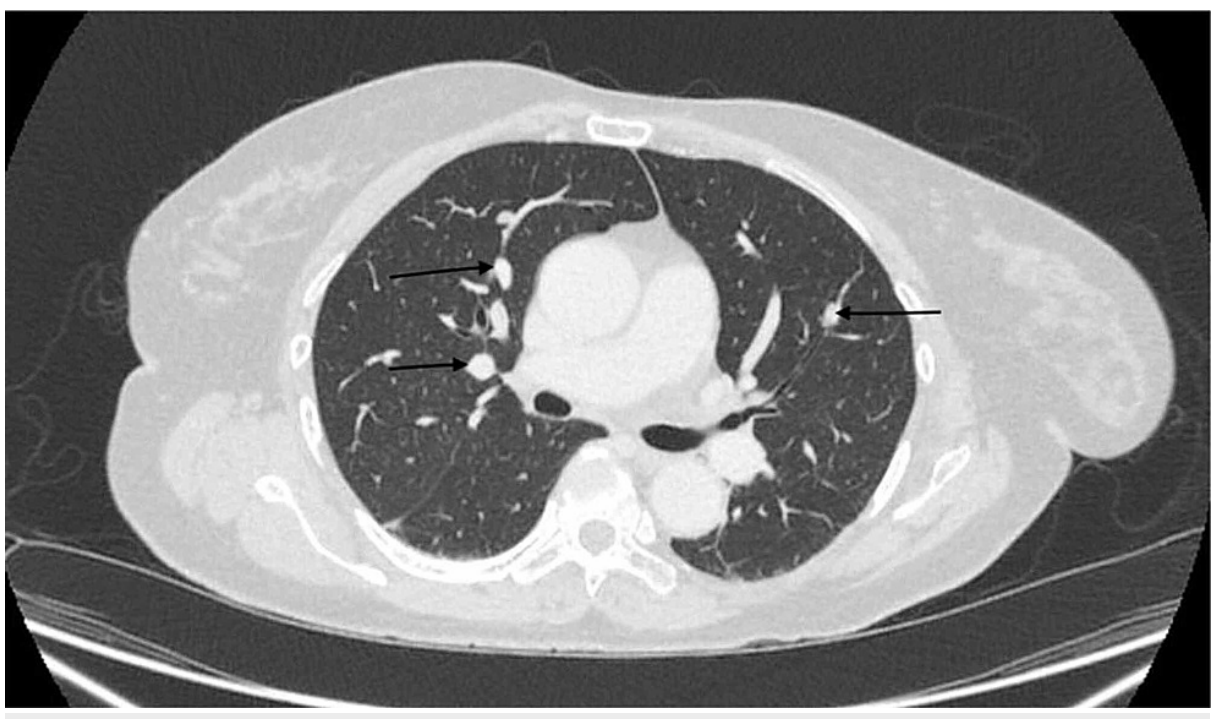

FIGURE 1: Computed tomography of chest showing multiple pulmonary nodules (black arrows) prior to starting everolimus $10 \mathrm{mg}$ PO.

mTOR Inhibitor, Everolimus

With the finding of metastatic disease, the patient was started on 10mg everolimus PO daily. Three weeks after treatment, the patient was hospitalized due to fever and headache, related to everolimus, and the dose was reduced to $5 \mathrm{mg}$ PO daily which was gradually uptitrated to $5 \mathrm{mg}$ daily in four weeks. CT scan two months after starting everolimus demonstrated marked interval enlargement of all pulmonary lesions seen on prior imaging, along with new lesions, indicative of progressive disease (Figure 2). Additionally, brain imaging performed for evaluation of dizziness showed a new enhancing lesion in the periphery of the left occipital lobe. Prior scans were negative for any intracranial lesion.

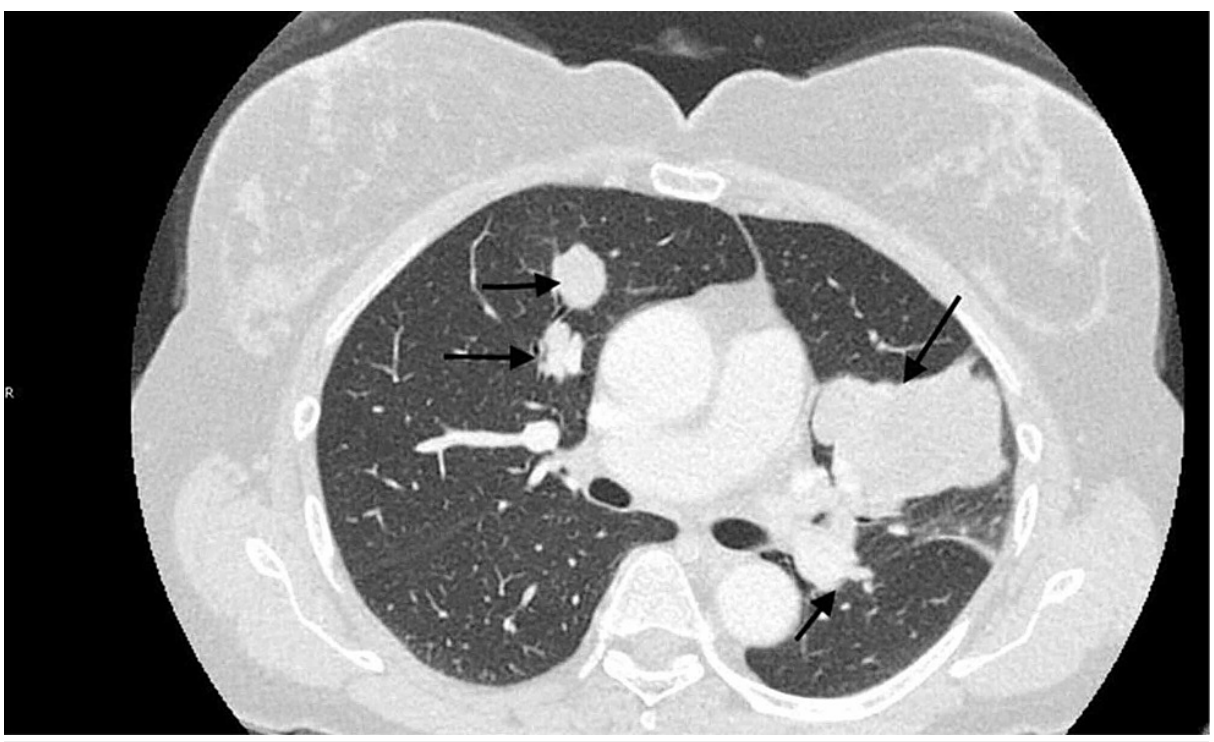

FIGURE 2: Computed tomography of the chest showing a significant progression of disease in lungs (black arrow) two months after starting everolimus and prior to starting nab-sirolimus.

Investigational mTOR Inhibitor, Nab-Sirolimus

After the failure of treatment with everolimus, the patient was enrolled in an expanded access protocol, PEX002 , with nab-sirolimus at $100 \mathrm{mg} / \mathrm{m}^{2}$ on day 1 and day 8 of a 21 -day cycle. She also received stereotactic radiosurgery to the metastatic lesion in her brain. The six-week restaging following two cycles of therapy 


\section{Cureus}

showed a marked decrease (50\%) in the target tumor lesion in her chest, indicating partial response, which was confirmed by the week 12 scans (Figure 3). The MRI brain also showed a reduction in the size of the cranial lesions.

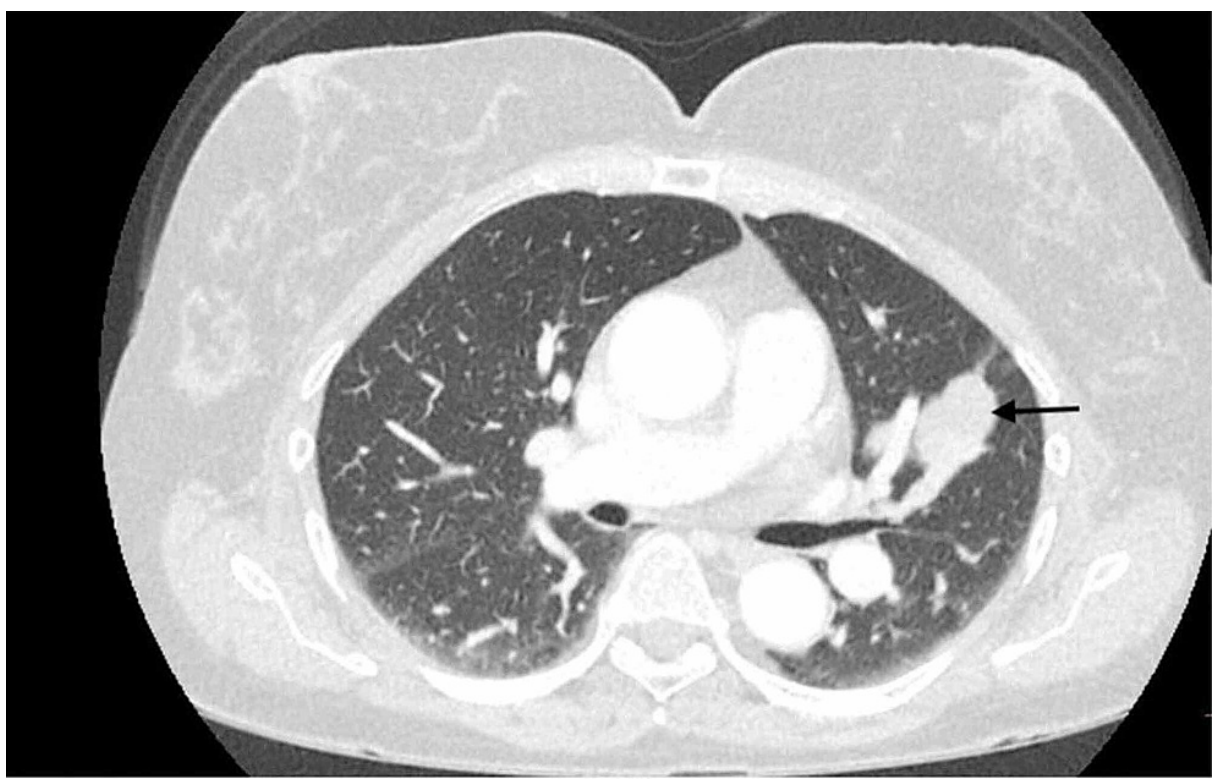

FIGURE 3: Computed tomography of chest showing a decrease in size of pulmonary nodules (black arrow) three months after starting nabsirolimus.

Clinical symptoms prior to nab-sirolimus included coughing-up blood, which ceased after two cycles, enabling her to run two miles without "getting winded." The patient developed grade 2

thrombocytopenia after cycle 2 for which the dose was reduced to $75 \mathrm{mg} / \mathrm{m}^{2}$. Other treatment-related adverse events were elevated lipids, maculopapular rash (grade 2), which were managed appropriately.

\section{Discussion}

PEComa is a rare form of sarcoma and has no effective therapy in the metastatic setting leading to a fatal outcome in most cases. Our patient has uterine PEComa, which is the most common site for PEComa's not associated with TSC and commonly behave as a high-grade sarcoma with distant metastases and death [4]. She also had a clinically aggressive disease based on size, >1mitoses/50 high power field.

Mutations in cytoplasmic tumor suppressor genes TSC1 or TSC2 are commonly seen in TSC [5]. Gene products of TSC1, TSC2 negatively regulates mTORC1 through inhibition of the mTOR kinase activator RHEB GTPase [6]. Thus, the absence of normally functioning TSC1/2 leads to increase mTOR activity, causing tumors to develop in various organ systems including the kidney, lung, brain and skin. Limited studies have demonstrated a similar mechanism of mTOR hyperactivation in non-TSC PEComas [7,8]. Cases published by Wagner et al., Italiano et al., Subbaiah et al. and Dickson et al. strongly support this model and demonstrate the use of mTOR inhibitors as a rational therapeutic target in patients with non-TSC PEComas. Some PEComa's are resistant to mTOR inhibitor treatment, despite optimal dosing and treatment as seen in our case. A few PEComas have a unique alveolar epithelioid rearrangement that harbor a TFE3 mutation and while these tumors have a normal expression of TSC2 they do not have TSC2 LOH [9]; however, this was not demonstrated in our patient and does not explain the cause for mTOR inhibitor resistance in all PEComa's and definitely is an area of future research. A combination of genetic findings in TSC1/TSC2 and staining for pS6-S235/236 may predict response to sirolimus/everolimus but we do not have enough data to support this.

The intriguing aspect about our patient is that even though she progressed through everolimus, she had a durable response to nab-sirolimus an albumin-bound sirolimus nanoparticle, also known as ABI-009 or nabrapamycin, an intravenous mTOR inhibitor. nab-Sirolimus utilizes an established nanoparticle albuminbound technology, resulting in a distinct PK profile, significantly higher anti-tumor activity, intra-tumoral drug accumulation, and mTOR target suppression $[10,11]$. This compound received Breakthrough Therapy Designation from the US Food and Drug Administration (FDA) in December 2018, for the treatment of patients with advanced (metastatic or locally advanced) malignant PEComa based on the AMPECT trial; these patients had no prior exposure to mTOR inhibitor. To our knowledge, this is the first patient who responded to nab-sirolimus after progressing through mTOR inhibitor. 


\section{Conclusions}

Cytotoxic chemotherapies for sarcoma show minimal benefit in PEComas and there are currently no drugs approved for this disease. Malignant PEComas frequently harbor mutations in the TSC1 and/or TSC2 genes that result in the activation of mTOR pathway. Therefore, mTOR signaling is a promising therapeutic target for malignant PEComa. NabSirolimus may therefore provide an important therapeutic option for these patients with locally invasive and metastatic PEComa beyond the currently available treatments and further research is definitely required in this area.

\section{Additional Information \\ Disclosures}

Human subjects: Consent was obtained or waived by all participants in this study. Conflicts of interest: In compliance with the ICMJE uniform disclosure form, all authors declare the following: Payment/services info: All authors have declared that no financial support was received from any organization for the submitted work. Financial relationships: All authors have declared that they have no financial relationships at present or within the previous three years with any organizations that might have an interest in the submitted work. Other relationships: All authors have declared that there are no other relationships or activities that could appear to have influenced the submitted work.

\section{References}

1. Martignoni G, Pea M, Reghellin D, Zamboni G, Bonetti F: PEComas: the past, the present and the future Virchows Arch. 2008. 452:119-32. 10.1007/s00428-007-0509-1

2. Kenerson H, Folpe AL, Takayama TK, et al.: Activation of the mTOR pathway in sporadic angiomyolipomas and other epithelioid cell neoplasms. Hum Pathol. 2007, 38:1361-71. 0.1016/j.humpath.2007.01.028

3. Dickson M, Ravi V, Riedel F, et al.: Weekly nab-sirolimus in patients with advanced malignant perivascular epithelioid cell tumors (EComa): results from AMPECT, an open-label phase 2 registration trial with independent radiology review. CTOS Annual Meeting. 2019, 3206452:

4. Fadare O: Uterine PEComa: appraisal of a controversial and increasingly reported mesenchymal neoplasm . Int Semin Surg Oncol. 2008, 5:7. 10.1186/1477-7800-5-7

5. The European Chromosome 16 Tuberous Sclerosis Consortium: Identification and characterization of the tuberous sclerosis gene on chromosome 16. Cell. 1993, 75:1305-15. 10.1016/0092-8674(93)90618-z

6. Kwiatkowski DJ, Thiele EA, Whittemore VH: Tuberous Sclerosis Complex. Wiley-VCH, Weinheim, Germany; 2010. 10.1002/9783527630073

7. Kenerson H, Folpe AL, Takayama TK, Yeung RS: Activation of the mTOR pathway in sporadic angiomyolipomas and other perivascular epithelioid cell neoplasms. Hum Pathol. 2007, 38:1361-71. 10.1016/j.humpath.2007.01.028

8. Pan CC, Chung MY, Ng KF, et al.: Constant allelic alteration on chromosome 16p (TSC2 gene) in perivascular epithelioid cell tumour (PEComa): genetic evidence for the relationship of PEComa with angiomyolipoma. J Pathol. 2008, 214:387-93. 10.1002/path.2289

9. Malinowska I, Kwiatkowski DJ, Weiss S, Martignoni G, Netto G, Argani P: Perivascular epithelioid cell tumors (PEComas) harboring TFE3 gene rearrangements lack the TSC2 alterations characteristic of conventional PEComas: further evidence for a biological distinction. Am J Surg Pathol. 2012, 36:783-4. 10.1097/PAS.0b013e31824a8a37

10. Gonzalez-Angulo AM, Meric-Bernstam F, Chawla S, et al.: Weekly nab-Rapamycin in patients with advanced nonhematologic malignancies: final results of a phase I trial. Clin Cancer Res. 2013, 19:5474-84. 10.1158/1078-0432.CCR-12-3110

11. Hou S, Schmid A, Desai N: Distinct pharmacokinetics, tissue distribution and CNS penetration of ABI-009 (nab-sirolimus). AACR. 2019, 79:3896. 10.1158/1538-7445.AM2019-3896 